\title{
Wörter im Zweifel. Ansätze einer linguistisch begründeten kritischen Semantik
}

\author{
Jörg Kilian (Braunschweig)
}

\begin{abstract}
Within the framework of pronunciation, morphology, and syntax, linguistic doubts and uncertainties belong to the everyday business and everyday life of linguists and public speakers. Cases of lexical semantic doubts, however, have been brushed aside by post-Saussurian and all the more by post-Bloomfieldian linguists so far. This paper deals with such lexical semantic doubts and uncertainties which not only rise from language use within "parole" but are caused by a conflict of opposite or even contradictory language norms of different "langue"varieties (e.g. the adjective positive as a medical term and in everyday colloquial language). Proceeding from an intensive discussion of the relationship between language use, language norms, and language system with special reference to Saussure, Hjelmslev, and Coseriu, the paper presents a theoretical approach to linguistically founded criticism of semantic norms by way of embedding lexical semantics into a framework of "Existenzweisen" (focusing on language use, norms, and system), "Existenzformen" (in particular norms of special discourses and varieties), and "language history and social stratification" (in particular age-based vocabularies and semantics). Finally, the paper turns this theoretic approach into practice by way of describing, explaining, and solving semantic doubts of the German word Zigeuner (gipsy). This example taken from the discourse on "political correctness" is investigated in the broader context of the word's historical, semantic, and pragmatic dimensions in different German discourses and varieties. The systematic relations between the various usages are presented in the form of a pragmatic and semantic network displaying the interconnections and borders between the meanings and gives recommendations to the word's use.
\end{abstract}

\section{Zweifelhafte Vagabunden: Zur Lexikologie des semantischen Zweifels}

Es gibt in der deutschen Sprache eine Klasse von Wörtern, die dazu tendieren, beim Überschreiten von Varietätengrenzen Verständigungs- und Kommunikationsprobleme zu evozieren. Dieser Befund ist empirisch nachweisbar, und er spiegelt sich mittelbar auch in einigen Publikationen der deutschen Sprachwissenschaft, die mit Titelstichwörtern wie "Wörterbuch der sprachlichen Zweifelsfälle" (Duden RgD), "Wörterbuch der Sprachschwierigkeiten" (Dückert/Kempcke), "Schwere Wörter" (Henne/Mentrup), "Brisante Wörter" (Strauß/Haß/Harras), "Kontroverse Begriffe" (Stötzel/Wengeler) auf derlei Wörter aufmerksam machen. Ein kritischer Punkt der sprachwissenschaftlichen Erforschung dieser Wörter ist es jedoch bislang, dass sie den Befund der Schwierigkeit, der Schwere, Brisanz und Kontro- 
verse beinahe ausschließlich kommunikations- und verständigungstheoretisch begründet. Als Kommunikationsstörung oder als Verständigungsproblem ist "Schwere" oder "Schwerverständlichkeit", sind "Brisanz" und "Kontroversen" eines Wortes allerdings ParolePhänomene, jeweils abhängig von der konkreten Kommunikationssituation und den konkreten Kommunikationsteilnehmern mit ihrem konkreten Wortschatzwissen. Dasselbe Wort mag in einer anderen Kommunikationssituation mithin "leicht" und unspektakulär sein (vgl. Strauß/Zifonun 1984: 388).

Der Befund des semantischen Zweifels hingegen bezieht sich nicht nur auf ein singuläres $\mathrm{Pa}-$ role-, sondern auf ein Langue-Phänomen, das heißt: Viele Wörter der deutschen Sprache wecken nicht erst situationsabhängig Zweifel, sondern haben diesen Zweifel gleichsam semantisiert; er ist dann als lexikalisch-semantisches Langue-Phänomen lexikologisch greifbar. Es handelt sich grundsätzlich um Wörter, die mit verschiedenen, möglicherweise gar oppositiven Teilbedeutungen bzw. mit verschiedenen Varianten ein und derselben Teilbedeutung in unterschiedlichen Varietäten- und Diskursnormen vertreten sind, beispielsweise um Wörter, die stereotypisch vagabundieren, wie etwa die Lexeme Mord und Totschlag, die in der juristischen Fachsprache prototypisch anders differenziert werden als in der Gemeinsprache.

Man kann deshalb zunächst festhalten:

1.) Lexikalisch gebundene semantische Zweifel sind begründet in Normenkonflikten innerhalb der semasiologischen oder/und onomasiologischen Struktur, in die ein Wort systematisch eingebunden ist. Semantische Zweifelsfälle sind deshalb Teilbedeutungszweifelsfälle. Wenn hingegen ein ganzes Lexem mit seinen semantischen, kommunikativ-pragmatischen und pragmatisch-stilistischen Potenzen standardsprachlich, fachsprachlich, gruppensprachlich oder sondersprachlich fest eingebunden ist (wie beispielsweise viele Fachwörter und Vulgarismen), dann ist es den Mitgliedern der Sprachgesellschaft bzw. den Mitgliedern der jeweiligen Sprechergruppen in diesen Eigenschaften bekannt und birgt keine lexikalisch gebundenen semantischen Zweifel.

2.) Ein semantischer Zweifelsfall als Normenkonflikt soll dadurch gekennzeichnet sein, dass ein Wort noch relativ unabhängig von einer konkreten ko- und kontextuellen Einbettung semantische - im weitesten Sinne - Zweifel hervorruft dahingehend, dass es, zum Beispiel, diskurs- oder varietätennormenabhängig soziale Missverständnisse evozieren könnte (wer das Verb türken i.S.v. 'Tatsachen vortäuschen' benutzt, muss in bestimmten Diskursen damit rechnen, als "xenophob", "türkische Mitmenschen gering schätzend" o.ä. angesehen zu werden); oder dass das Wort falsche, unerwünschte bzw. gesellschaftlich oder gruppenspezifisch negativ sanktionierte "Weltansichten" konzeptualisieren könnte (wer das Wort Fräulein als Anrede benutzt, muss in bestimmten Diskursen damit rechnen, als "konservativ", "Kritiker der Geschlechtergleichstellung" o.ä. eingeschätzt zu werden).

Ich möchte das dahingehend konkretisieren, dass all diese Fälle des semantischen Zweifels darauf zurückzuführen sind, dass eine Wortbedeutung oder eine Variante einer Wortbedeutung eines (auch) der Standardsprache angehörenden Lexems sich ins Periphere, Besondere, Seltene (vgl. Hausmann 1990) anderer Varietäten und besonderer Diskurse begibt (et vice versa) und dadurch einen Normenkonflikt heraufbeschwören kann. 


\section{Semantische Existenzweisen und Existenzformen eines Wortes}

Wenn semantische Zweifelsfälle auf Normenkonflikte innerhalb der semasiologischen oder/und onomasiologischen Struktur eines Wortes zurückzuführen sind, dann betreffen sie das Sprachzeichen weder als ganzes Lexem auf der Ebene des virtuellen Sprachsystems noch als aktualisiertes Wort auf der Ebene des aktuellen Sprachgebrauchs, denn das Sprachsystem ist über (fast) jeden Zweifel erhaben, und der Sprachgebrauch kennt nur situationsabhängige individuelle Zweifelsfälle. Lexikologische Erklärungen des semantischen Zweifels müssen deshalb davon ausgehen, dass die semantischen Zweifel, die von einem Wort ausgelöst werden können, der Wortbedeutung im Laufe ihrer kultur- und sozial-, mentalitäts- und ideengeschichtlichen Formung irgendwo zwischen virtuellem Sprachsystem und aktuellem Sprachgebrauch beigegeben wurden. Es bedarf deshalb einer Sprach- (und Bedeutungs-) Theorie, die die Historizität der Sprache und die Heterogenität der Sprachgesellschaft nicht erst im realisierten Sprachgebrauch, sondern bereits im Rahmen des Konstrukts der virtuellen Existenzweisen von Sprache berücksichtigt. Auch aus diesem Grund ist Ferdinand de Saussures Identifikation einer (bzw. der Standard-) Norm mit einem mehr oder minder homogenen Sprachsystem (vgl. Saussure 1967: 11) bedeutungstheoretisch ebenso unergiebig wie die aus Ludwig Wittgensteins lakonischer Äußerung, die Bedeutung eines Wortes sei sein Gebrauch in der Sprache (Wittgenstein, PU §43) herausgelesene Auflösung der lexikalischen Bedeutung in eine unergründliche Zahl von Einzelgebräuchen. Erst im Rahmen einer Sprach- (und Bedeutungs-) Theorie, die aus der Heterogenität der Sprachgesellschaft verschiedene, untereinander in ständiger Wechselwirkung befindliche virtuelle Teilsysteme, nämlich Varietätenund Sprachverkehrsnormen ableitet, wird die Existenz semantischer Zweifelsfälle sprachtheoretisch nicht nur möglich, sondern mithin (auch für den Bedeutungswandel) gar notwendig - man denke beispielsweise an die Gegensatzsemantik von positiv in der Alltags- und in der medizinischen Fachsprache oder an die Semantik von Walfisch, die enzyklopädisch gerade das Merkmal [- FISCH] enthalten muss. Es ergibt sich folgendes Modell der Existenzweisen von Sprache:

Modell der Existenzweisen von Sprache (variiert aus: Kilian 2001)

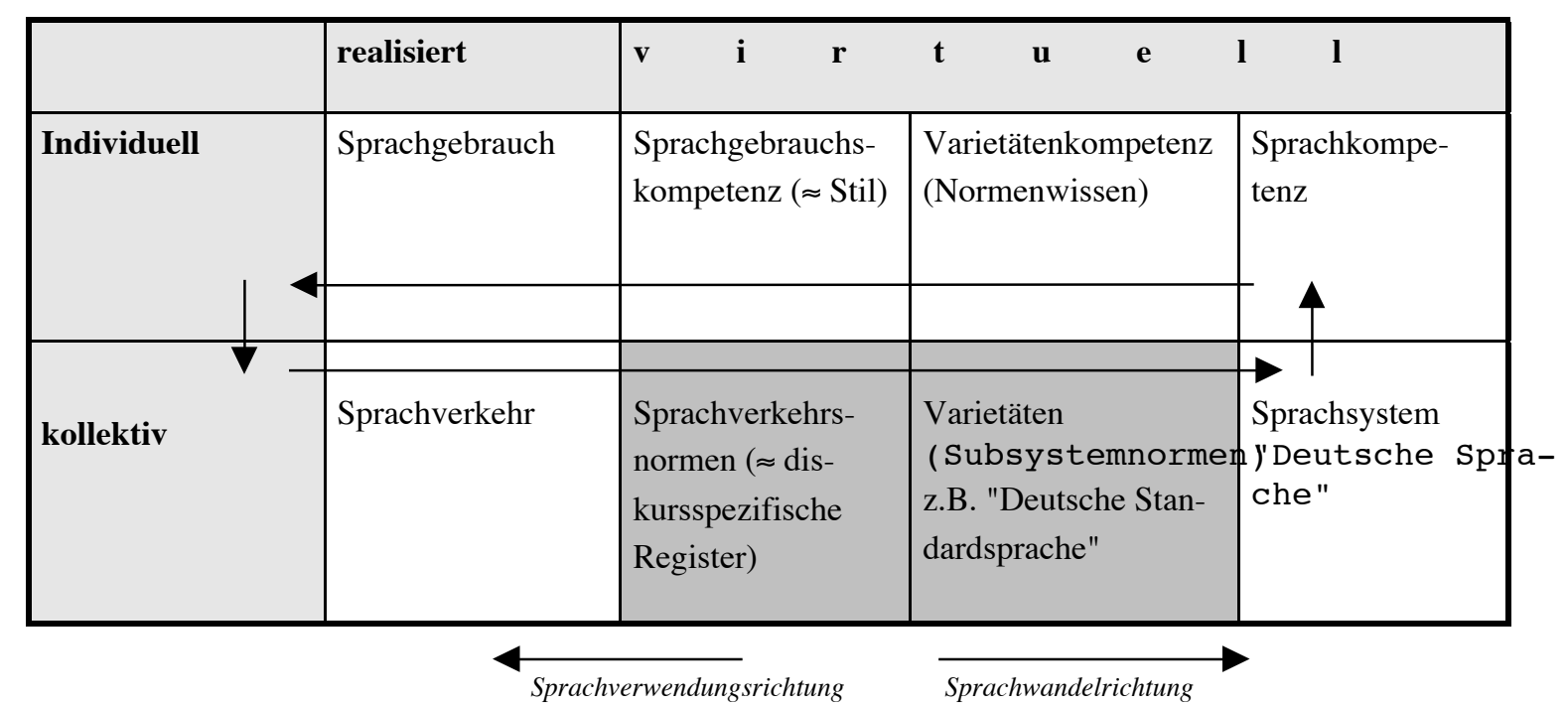

ISSN 1615-3014 
Es gibt demnach nicht nur die zwei Existenzweisen von Sprache, die als Sprachsystem und Sprachgebrauch bekannt sind, sondern zwischen ihnen weitere Existenzweisen, die, insofern sie Auswahlen aus den Möglichkeiten des Systems darstellen, als je besonders normativ gebundene Subsysteme zu begreifen sind. Dabei darf "normativ" nicht allein in dem Sinne verstanden werden, dass es sich um kodifizierte, statuierte Normen handelt, sondern um historisch von verschiedenen Sprechergruppen für deren jeweilige gesellschaftlichen Kommunikations- und Praxisbereiche etablierte Konfigurationen konventioneller Regeln, die für die Ausgliederung von Existenzformen verantwortlich sind. Aus diesem Grund tritt, als drittes, die Historizität der Sprache, die Sprachgeschichte, hinzu, die in Bezug auf die semantischen Zweifelsfälle als Sprachgeschichte der Mitlebenden zu konzipieren ist. Im Modell der Existenzweisen und Existenzformen ist diese historische Dimension durch die Angabe der "Sprachverwendungsrichtung" angezeigt, die stets vom Sprachsystem bzw. der individuellen Sprachkompetenz über den Filter der Normen zum Sprachverkehr bzw. zum Sprachgebrauch verläuft und auf die Normen, mithin gar das System zurückwirkt ("Sprachwandelrichtung"). Dementsprechend tritt zwischen die beiden sprachtheoretischen Extrempositionen des polysemen virtuellen Lexems einerseits und des monosemierten aktuellen Wortes andererseits die (virtuelle) Norm bzw. treten die (virtuellen) Varietäten- und Diskursnormen, die die Aktualisierung eines Lexems steuern.

In Anlehnung an die sprachtheoretischen Entwürfe von Louis Hjelmslev und Eugenio Coseriu (vgl. Kilian 2001: 305ff.) lässt sich die Ebene der Normen als der lexikologische Entstehungsort des semantischen Zweifels wie folgt erklären: Sprachnormen sind, wie Eugenio Coseriu es formuliert, je spezifische "Systeme von obligatorischen Realisierungen der sozialen und kulturellen Auflagen" (Coseriu 1971: 69). Dabei darf die Platzierung der "Sprachnorm" zwischen "Sprachsystem" und "Sprachverkehr" nicht darüber hinwegtäuschen, dass Sprachnormen virtuelle Existenzweisen von Sprache sind; Coserius Verortung der "Norm" sowohl auf der Langue- wie auf der Parole-Seite ist daher nicht erklärungskräftig. Louis Hjelmslevs Explikation des Saussure'schen Langue-Begriffes ist ihr deshalb vorzuziehen: Für Hjelmslev ist die Saussure'sche Langue als dreiteilig anzusehen (die Parole kommt dann als realisierte Existenzweise hinzu), nämlich 1.) "als eine reine Form, unabhängig von ihrer sozialen Realisiation und ihrer materiellen Manifestation definiert" ("une forme pure": "schéma"), 2.) "als eine materielle Form, definiert durch eine soziale Realisation, aber unabhängig noch vom Detail der Manifestation" ("une forme materielle": "norme") und 3.) "als eine einfache Summe von in einer gegebenen Gesellschaft angenommenen Gewohnheiten, die durch beobachtete Manifestationen definiert sind" ("un simple ensemble des habitudes": "usage"). ${ }^{1}$ Von der kollektiven Langue bis hin zur individuellen Parole gibt es bei ihm also einen systematischen Weg der Konkretion: "schéma" - "norme" - "usage" - "acte", adaptiert im oben stehenden Modell als (kollektiv) "Sprachsystem" - "Varietätennormen" - "Sprachverkehrs-

\footnotetext{
${ }^{1}$ Vgl. Hjelmslev 1942/1974: 47; dazu Maier 1984: 10ff., deren Arbeit auch die originalen französischen Termini entnommen sind. Dass Hjelmslev in Bezug auf den Begriff des "Sprachgebrauchs" schillernd bleibt und, einerseits, den "Sprachgebrauch" als "Summe von Gewohnheiten" und als "usus" bezeichnet, ihn andererseits aber schließlich doch ganz als Entsprechung der Saussure'schen Parole hinstellt (Hjelmslev 1942/1974: 47 u. 55; vgl. auch Hjelmslev 1963/1968: 47 u. 54), braucht hier nicht weiter verfolgt zu werden.
} 
normen" - "Sprachverkehr" bzw. (individuell) "Sprachkompetenz" - "Varietätenkompetenz" "Sprachgebrauchskompetenz" - "Sprachgebrauch".

Die für eine linguistische Kritik der semantischen Zweifelsfälle wichtigsten Existenzweisen sind "norme" und "usage", hier als Varietätennormen und Sprachverkehrsnormen benannt. Varietätennormen sind "für konkrete gesellschaftliche Praxis- und Kommunikationsbereiche etablierte Konfigurationen konventioneller Regeln [...], die aufgrund der jeweils spezifischen außersprachlichen Bedingungen der Kommunikation die Auswahl sprachlicher Mittel und deren Gebrauch in diesen Kommunikationsbereichen steuern." (Kilian 1997: 64). Varietätennormen stellen spezifische, die Heterogenität der Sprachgesellschaft spiegelnde Auswahlen aus dem Sprachsystem dar, führen zur Herausbildung von Subsystemen - eben den Varietäten - und umgrenzen die Sprachverkehrsnormen. Demgegenüber sind Sprachverkehrsnormen nicht nur durch die Varietätennormen eingegrenzt, sondern zusätzlich durch Diskursund Situationstypen bedingt, die im Rahmen von Varietätennormen für je bestimmte Anlässe des Sprachgebrauchs noch bestimmtere Auswahlen erforderlich machen und dabei je spezifische "Kollektivgewohnheiten" und "Lebensformen" einfließen lassen. Im Rahmen des kollektiven Teilbereichs handelt es sich um systematische Konfigurationen konventioneller Regeln, die den Realisierungen der Varietäten zugrunde liegen, sozial verbindlich sind und als "Register" gefasst werden können.

Aus den angedeuteten Gründen sind weder die im engeren Sinne strukturalistischen noch die im engeren Sinne pragmalinguistischen Semantiktheorien für eine kritische Semantik sehr ergiebig. Denn eine Lexikologie des semantischen Zweifels muss über die minimaldistinktive Systembedeutung und über die je konkrete Gebrauchsbedeutung hinaus gerade das heterogen prototypisch, stereotypisch und assoziativ geronnene Bedeutungspotential eines Wortes ermitteln, zu dem sich lexikalisch nicht geronnene assoziative Stereotype gesellen, die Vorurteile, die Mythen, die Denkbräuche, das vermeintliche Weltwissen, das sich um Wortbedeutungen und Wortgebräuche rankt und sehr oft in Phraseolexemen und Komposita unbeachtet weiterlebt und tradiert wird: Man denke beispielsweise an das Wort blond, dem in gewissen Sprachverkehrsnormen das assoziierte Stereotyp 'dumm' beigelegt wird, oder man denke an die assoziative Semantik der auch in standardsprachlichen Wörterbüchern noch gebuchten Redensart Zustände wie in einer Judenschule, die abwertende Stereotype über Synagogen tradiert.

Erfolg versprechender als die im engeren Sinne strukturalistischen und pragmalinguistischen Bedeutungstheorien sind deshalb semantiktheoretische Ansätze, die lexikalische Bedeutung von vornherein in ihrer historischen varietäten- und diskursspezifischen Gewichtung erfassen und dadurch die Heterogenität der Sprachgesellschaft semantiktheoretisch einlösen. Es sind dies mithin Ansätze, die von einer Semantik der distinktiven Merkmale abrücken und viel stärker die so genannten enzyklopädischen, sodann aber auch die noch nicht lexikalisierten assoziativen Merkmale, also die Stereotype im engeren Sinne, einbeziehen. Vornehmlich zu nennen sind hier die Prototypen- und Stereotypensemantik sowie - wegen der Berücksichtigung epistemischer (bzw. enzyklopädischer) und assoziativer Beziehungen - die FrameSemantik. 


\section{$3 \quad$ Zur Kritik des Zweifels - am Beispiel Zigeuner}

Ich veranschauliche dies abschließend an einem Beispiel, das ich an anderer Stelle ausführlicher beschrieben habe (vgl. Kilian 2003a): Das "Große Wörterbuch der deutschen Sprache" aus dem Duden-Verlag, 3. Aufl. 1999, erklärt die Bedeutung des Lemmas Zigeuner wie folgt:

Zilgeulner, der; -s, - [spätmhd. ze-, ziginer, H. u.]: 1. Angehöriger eines über viele Länder verstreut lebenden, meist nicht sesshaften u. mit Wohnwagen o.Ä. umherziehenden Volkes (wird von den Betroffenen selbst oft als abwertend empfunden; vgl. ${ }^{2}$ Rom, Sinto). 2. (ugs., meist abwertend) jmd., der ein unstetes Leben führt, wie ein Zigeuner (1) lebt [...]." ( ${ }^{3} \mathrm{GWb} 10,1999$, 4634).

Das Wort Zigeuner wird hier ausschließlich als "abwertende" Personenbezeichnung geführt, allerdings ohne dass eine Empfehlung für seinen Gebrauch gegeben wird. Zwei Teilbedeutungen werden erklärt, einmal die Bezeichnung für Menschen einer ethnischen Minderheit und sodann eine davon abgeleitete allgemeinere Teilbedeutung.

Das Wort Zigeuner hat allerdings noch eine dritte Teilbedeutung, die hier gar nicht erwähnt wird. Im DWb wird sie als "romantische sicht", im "Deutschen Wörterbuch" von Hermann Paul als "im Bereich von Kunst und Literatur [...] romantisierend" beschrieben und u.a. mit den enzyklopädischen Merkmalen "ungebundene lebensweise", "malerische kleidung" und "musizieren" versehen. Heute spielt sie etwa noch eine Rolle, wenn in Katalogen "Zigeunerkostüme" für die Faschingszeit beworben werden. Dass diese Teilbedeutung im zehnbändigen Duden aus dem Jahr 1999 nicht eigens vermerkt ist, verwundert denn auch, gehört sie doch der deutschen Gegenwartssprache an, wie man selbst diesem "Großen Wörterbuch der deutschen Sprache" entnehmen kann, wenn man u.a. die Lemmata Zigeunerkapelle und Zigeunermusik dort gebucht findet - vom Zigeunerschnitzel ganz zu schweigen, das wohl kaum die stereotypen Vorstellungen von "nicht sesshaftem", "unstetem" Leben im "Wohnwagen" wecken soll.

\section{Gegenwartssprachlich-semasiologisches Feld Zigeuner (nach ${ }^{3} \mathrm{GWb}$ und ${ }^{10} \mathrm{Paul}$ )}

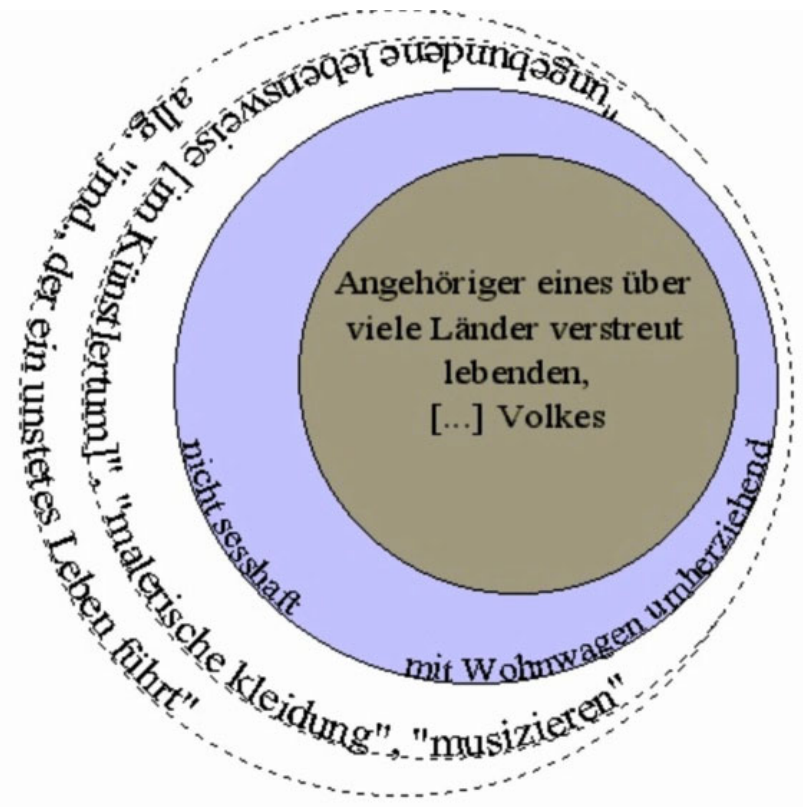


Es gibt gegenwartssprachlich demnach folgende drei Teilbedeutungen, die den Begriff "Zigeuner" denotativ je unterschiedlich, in Bezug auf die konnotative und mithin deontische Bedeutung gar gegensätzlich konzeptualisieren. Schon daraus müssen sich, folgt man der eingangs formulierten These, semantische Zweifelsfälle ergeben:

1. "Angehöriger eines über viele Länder verstreut lebenden, meist nicht sesshaften u. mit Wohnwagen o. Ä. umherziehenden Volkes" ( $\left.{ }^{3} \mathrm{GWb}\right)$;

2. ("romantisierend"): jmd., der eine ungebundene (nicht-bürgerliche) Lebensweise im Bereich des Künstlertums führt (z.B. $\left.{ }^{10} \mathrm{Paul}\right)$;

3. "(ugs. [...]) jmd., der ein unstetes Leben führt, wie ein Zigeuner (1) lebt [...]." ( $\left.{ }^{3} \mathrm{GWb}\right)$.

Den größten Zweifel erregt in der deutschen Gegenwartssprache die erste Teilbedeutung, die wohl als "Hauptbedeutung" (im Sinne Hermann Pauls) gelten darf. Diese Hauptbedeutung dient der Benennung von Menschen einer bestimmten Ethnie. Als standardsprachlich prototypische Merkmale, die alle Vertreter dieser Personengruppe nach Ausweis des ${ }^{3} \mathrm{GWb}$ aufweisen müssten, werden, neben dem auf Menschen verweisenden Genus proximum "Angehöriger eines über viele Länder verstreut lebenden [...] Volkes", die spezifisch differenzierenden enzyklopädischen Merkmale "nicht sesshaft", "mit Wohnwagen umherziehend" sowie die Stilmarkierung "abwertend" angeführt. Diese Merkmale leiten demnach die Konzeptualisierung des Begriffs "Zigeuner" und die Kategorisierung bestimmter Menschen zu Vertretern der Kategorie Zigeuner in der deutschen Gegenwartssprache.

Prototypische "Hauptbedeutung" von Zigeuner und assoziierte Stereotype (nach DWb, Wehrle/Eggers, ${ }^{3} \mathrm{GWb}$ und ${ }^{10} \mathrm{Paul}$ )

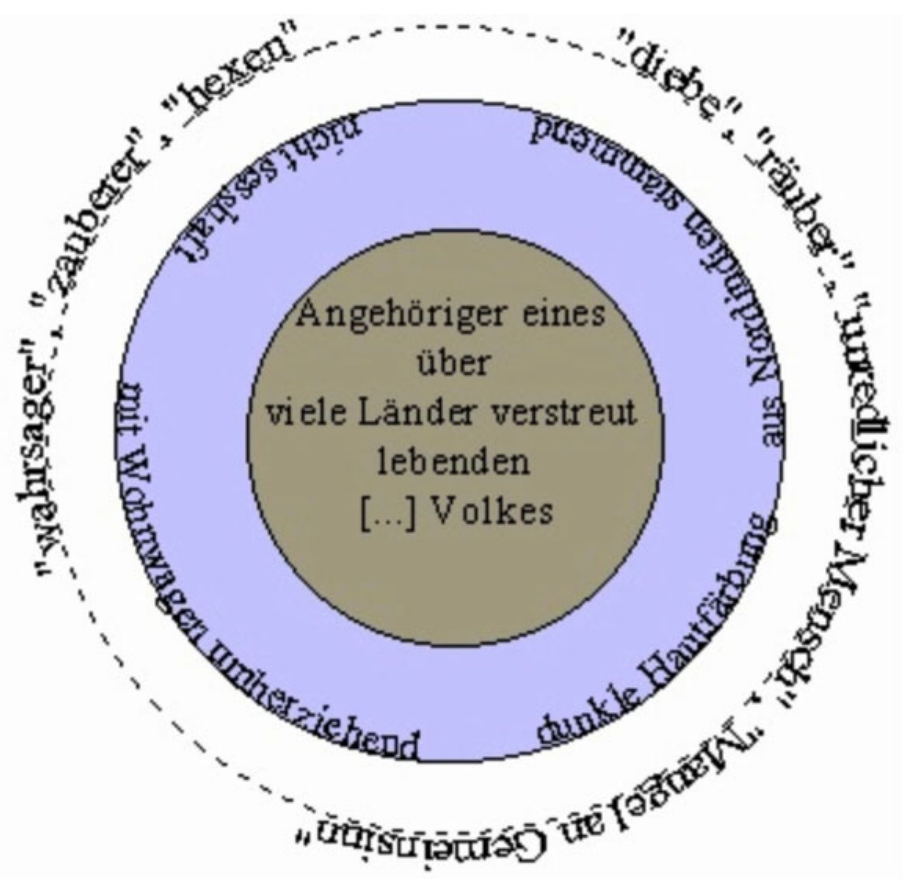


Hinzu kommen die im Laufe der Sprachgeschichte an dieses Wort gehefteten, zum Teil schon lexikalisierten, zum Teil nur kollektiv assoziierten Stereotype. Ein Stereotyp ist, nach Putnam, "eine konventional verwurzelte (häufig übelmeinende und möglicherweise völlig aus der Luft gegriffene) Meinung [idea] darüber, wie ein X aussehe oder was es tue oder sei" (Putnam 1990: 68). Nicht alle Stereotype sind semantisiert und damit lexikalisch fest gebunden; in vielen Fällen (wie z.B. beim oben genannten blond) bleiben die Stereotype lediglich assoziiert, allerdings kollektiv assoziiert bei einem großen Teil der Mitlebenden. Die großen historischen Wörterbücher des Deutschen, der "Grimm" (DWb) und der "Paul", führen neben den denotativen und konnotativen Merkmalen auch solche assoziierten Stereotype an, der "Grimm" etwa die zur Bedeutungserklärung herangezogenen Prädikate "diebe", "räuber", "wahrsager", "zauberer", "hexen" (DWb 31, 1956: 1257ff.), die das Wort Zigeuner in den Peripherien verschiedener Synonymenfelder verorten. Desgleichen hat der "Wehrle-Eggers", das 1993 unverändert nachgedruckte große deutsche Synonymenwörterbuch, das ein "Wegweiser zum treffenden Ausdruck" sein will, das Stichwort Zigeuner in die Peripherien unterschiedlicher prototypischer Felder eingeordnet, zum Beispiel in das Wortfeld zu "Landfahrer", wo es mit Nomade und Beduine, Tater (niederdeutsch: 'Tatar') und Wandervolk einen relativ zentralen Platz erhält; Zigeuner taucht im selben Wörterbuch aber auch noch in anderen Wortfeldern auf, nämlich im Wortfeld "Mangel an Gemeinsinn", wo es zwischen Bagage und Bettelvolk, Volksfeind und Volksbetrüger zu stehen kommt, und schließlich noch einmal im Wortfeld "unredlicher Mensch", wo es in die Reihe von Schlawiner und Rastelbinder, Schwindler und Schwindelhuber eingereiht ist. Die movierte Form Zigeunerin erhält hier einen eigenen Eintrag, nämlich im Wortfeld "Wahrsager", dort allerdings erst in der äußeren Peripherie, am Schluss der Reihe nach Kartenlegerin und Hexe.

Nun könnte man allein schon aufgrund der Tatsache, dass die angeführten enzyklopädischen Merkmale, also die vom "nicht sesshaften" Leben im "Wohnwagen", für viele Sinti und Roma zu Beginn des 21. Jahrhunderts gar nicht zutreffen, dass also Wort und Welt einander nicht mehr korrespondieren, eine Kritik der semantischen Normen begründen und das Wort, wie es die "political Correctness" verlangt, aus dem Wortschatz zu tilgen versuchen. Es bedarf überdies keiner besonderen Begründung, dass Sinti und Roma sich gegen diese assoziierten und teils lexikalisierten diskriminierenden Stereotype, die dem Wort Zigeuner anhaften, zur Wehr setzen müssen.

Ganz so einfach, wie es ein Wortvermeidungsverbot suggeriert, ist es indes nicht, denn semantische Zweifelsfälle sind wesentlich komplexer strukturiert als die semantisch, stilistisch und pragmatisch relativ klaren Fälle etwa des obszönen und vulgären Wortschatzes. Ich greife noch einmal das Modell der Existenzweisen von Sprache auf, um die komplexe diskurs- und varietätensemantische Struktur von Zigeuner im Ausriss zu veranschaulichen: 
Ansätze einer linguistisch begründeten kritischen Semantik

\begin{tabular}{|c|c|c|c|c|}
\hline & realisiert & i & $\mathbf{t}$ & I \\
\hline Individuell & Sprachgebrauch & $\begin{array}{l}\text { Sprachgebrauchs- } \\
\text { kompetenz }(\approx \text { Stil) }\end{array}$ & $\begin{array}{l}\text { Varietätenkompetenz } \\
\text { (Normenwissen) }\end{array}$ & Sprachkompetenz \\
\hline kollektiv & Sprachverkehr & $\begin{array}{l}\text { Sprachverkehrs- } \\
\text { normen ( } \approx \text { dis- } \\
\text { kursspezifische } \\
\text { Register), z.B. } \\
\text { öffentlich-politische } \\
\text { Diskurse, z.B. "Mi- } \\
\text { grationsdis-kurs" } \\
\text { oder "Diskurs um } \\
\text { Wiedergutma- } \\
\text { chung der NS- } \\
\text { Verbrechen": in } \\
\text { referierendem Ge- } \\
\text { brauch wird Zigeu- } \\
\text { ner meist, in kon- } \\
\text { kreten Anreden stets } \\
\text { durch Sinti und } \\
\text { Roma ersetzt; } \\
\text { in historisch- } \\
\text { politischen Diskur- } \\
\text { sen, z.B. über NS- } \\
\text { Verbrechen in refe- } \\
\text { rierendem Gebrauch } \\
\text { meist nicht ersetzt } \\
\text { (vgl. z.B. "Enzyklo- } \\
\text { pädie des Holo- } \\
\text { caust", }{ }^{2} 1998,1630 \text { ); } \\
\text { in umgangssprach- } \\
\text { lich-referierendem } \\
\text { Gebrauch auch in } \\
\text { der standardsprachli- } \\
\text { chen Tb1 und ihren } \\
\text { assoziierten Stereo- } \\
\text { typen meist nicht } \\
\text { ersetzt; } \\
\text { in Titeln (Strauß: } \\
\text { "Der Zigeunerba- } \\
\text { ron"; Alexandra: } \\
\text { "Zigeunerjunge") } \\
\text { und Produktnamen } \\
\text { ("Zigeunerschnit- } \\
\text { zel") nicht ersetzt. }\end{array}$ & $\begin{array}{l}\text { Varietäten } \\
\text { (Subsystemnormen), } \\
\text { z.B. } \\
\text { Standardsprache: 1.) } \\
\text { i.S.v. 'Mitglied einer } \\
\text { ethnischen Minderheit': } \\
\text { "abwertend", meist } \\
\text { ersetzt; 2.) i.S.v. "unge- } \\
\text { bundene lebensweise } \\
\text { [im Künstlertum]" } \\
\text { (DWb): positiv konno- } \\
\text { tiert. In Komposita } \\
\text { (Zigeunerleben, -musik } \\
\text { u.a.) unterschiedliche } \\
\text { Konzeptualisierungen } \\
\text { (kaum ersetzbar); } \\
\text { Umgangssprache: allg. } \\
\text { 'jmd., der viel reist o. } \\
\text { unstet lebt'; unter- } \\
\text { schiedliche Konnotatio- } \\
\text { nen; mitunter auch als } \\
\text { Schimpfwort gebraucht } \\
\text { (mit unterschiedlichen } \\
\text { assoziierten Merkma- } \\
\text { len); } \\
\text { Fachsprache: 'im 10. } \\
\text { Jh. aus Nordindien } \\
\text { ausgewanderte ethni- } \\
\text { sche Gruppierung' (so } \\
\text { z.B. in Zigeunerspra- } \\
\text { che); } \\
\text { in der Literaturspra- } \\
\text { che oft wegen des } \\
\text { "romantisierenden" } \\
\text { Tons nicht ersetzt; } \\
\text { in der Mediensprache } \\
\text { diskursabhängig ver- } \\
\text { wendet. }\end{array}$ & $\begin{array}{l}\text { Sprachsystem } \\
\text { "Deutsche Spra- } \\
\text { che" }\end{array}$ \\
\hline
\end{tabular}


Es ist unmittelbar einsichtig, dass selbst die Hauptbedeutung des Wortes Zigeuner in unterschiedlichen Varietäten- und Sprachverkehrsnormen unterschiedliche Varianten entfaltet, die die in der standardsprachlichen Varietätennorm und in politolektalen Sprachverkehrsnormen normativ gefasste "abwertende" Semantik so nicht teilen, zumindest variieren. Die meisten Mitlebenden - und zwar nicht nur die älteren - führen das Wort Zigeuner in ihren umgangssprachlichen Sprachverkehrsnormen nach wie vor für die Menschen, die im öffentlich-politischen Diskurs mit allem Recht Sinti und Roma genannt sein wollen. Dieser Gebrauch tradiert überkommene Stereotype; er ist wohl auch Ausdruck xenophober Einstellung, er ist aber nicht grundsätzlich "ein Akt rassistischer Gewalt", wie Karsta Frank meint (Frank 1996: 214f.). Ob mit diesem Gebrauch in jüngster Zeit ein politisch korrektes Unbehagen einhergeht, indem etwa die Normen des öffentlich-politischen Diskurses auf die umgangssprachlichen Sprachverkehrsnormen einwirken, ist den Daten noch nicht zu entnehmen. Bemerkenswert ist in diesem Zusammenhang allerdings, dass eine von Betroffenen selbst gepflegte Homepage den Namen www.zigeuner.de trägt. Und weiter: Am Abend des 16. August 2002 wird im ZDF-Magazin "Aspekte" ein Beitrag über Leni Riefenstahls zwielichtige Rekrutierung von KZ-Häftlingen für ihren 1940 gedrehten Film "Tiefland" mediensprachlich mit den Worten "Schicksal eines Zigeunermädchens" eingeleitet; in der Sprachgermanistik ist das Wort Zigeunersprache fachsprachlicher Terminus für 'Romani' (vgl. Siegmund A. Wolf: Großes Wörterbuch der Zigeunersprache, $\left.{ }^{2} 1987\right)$; in Günter Grass' Novelle "Im Krebsgang" bekennt der Erzähler literatursprachlich nicht ohne Stolz: "ich soll ja [...] von echten Zigeunern abstammen" (211).

\section{$4 \quad$ Schluss}

Die kultur- und sozial-, ideen- und mentalitätsgeschichtlichen "Weltansichten", die in der semasiologischen und onomasiologischen Struktur des Wortes Zigeuner lexikalisiert oder mit ihr assoziativ verknüpft sind, dürfen als Ursachen für die abwertende Semantik haftbar gemacht werden. Die semantischen Zweifel jedoch, die dieses Wort heute aufwirft, sind begründet in der polysemen Struktur und den unterschiedlichen, miteinander konfligierenden Varietäten- und Sprachverkehrsnormen. Die "abwertende" Semantik einer Teilbedeutung, und sei es der Hauptbedeutung, führt allein nämlich nicht zum Zweifel, denn sie ist zweifellos "abwertend".

Zusammenfassend ist festzuhalten, dass es im deutschen Wortschatz Wörter gibt, die zwar noch nicht auf der Ebene des Sprachsystems, aber auch nicht erst auf der Ebene des konkreten Sprachgebrauchs Probleme bereiten und Zweifel aufwerfen, sondern durch das Zusammenspiel verschiedener virtueller Varietäten- und Sprachverkehrsnormen mit einer zweifelhaften semantischen Potenz ausgestattet sind. Als wichtigste Ursache für Normenkonflikte lassen sich unterschiedliche varietäten- und diskursspezifische Konzeptualisierungen anführen, die in Form von Teilbedeutungen und Gebrauchsregeln lexikalisch gebunden werden und für die Mitglieder einer Sprechergruppe normativ bindend sind. Die drei sprachtheoretisch begründeten und im oben stehenden Modell veranschaulichten kategoriellen Kriteriengruppen: Existenzweisen, Existenzformen und Sprachgeschichte bilden die sprachwissenschaftliche Grundlage für eine kritische Beschreibung der semantischen Normen; eine 
Beschreibung, die sich des semantischen Zweifelsfalls annimmt und operationalisierbare lexikologische Kriterien für die Auffindung von "Wörtern im Zweifel" bereitstellt.

\section{Literaturangaben}

\section{Wörterbücher:}

Dückert, Joachim/Kempcke, Günter: Wörterbuch der Sprachschwierigkeiten. Zweifelsfälle, Normen und Varianten im gegenwärtigen deutschen Sprachgebrauch. Leipzig 1984, 3., durchgesehene Aufl. 1989.

Duden RgD: Duden. Richtiges und gutes Deutsch. Wörterbuch der sprachlichen Zweifelsfälle. 4. Aufl. hrsg. und bearb. vom Wissenschaftlichen Rat der Dudenredaktion. Mannheim usw. 1997. 5. Aufl. Mannheim usw. 2001.

DWb: Deutsches Wörterbuch von Jacob Grimm und Wilhelm Grimm. 16 Bde. in 32 Teilbdn. Leipzig 1854-1960. Ndr. München 1984.

${ }^{3}$ GWb: DUDEN. Das große Wörterbuch der deutschen Sprache in zehn Bänden. 3., völlig neu bearb. und erw. Aufl., Mannheim usw. 1999.

Stötzel, Georg/Wengeler, Martin: Kontroverse Begriffe. Geschichte des öffentlichen Sprachgebrauchs in der Bundesrepublik Deutschland. Berlin, New York 1995.

Strauß, Gerhard/Haß, Ulrike/Harras, Gisela: Brisante Wörter von Agitation bis Zeitgeist. Ein Lexikon zum öffentlichen Sprachgebrauch. Berlin, New York 1989.

Wehrle-Eggers: Deutscher Wortschatz. Ein Wegweiser zum treffenden Ausdruck. Stuttgart 1961, Ndr. Stuttgart, Dresden 1993.

\section{Forschungsliteratur:}

Coseriu, Eugenio (1971): "System, Norm und Rede". In: ders.: Sprache. Strukturen und Funktionen. XII Aufsätze zur allgemeinen und romanischen Sprachwissenschaft. Uwe Petersen (ed.). 2. verb. Aufl. Tübingen (zuerst 1969).

Frank, Karsta (1996): "Political Correctness: Ein Stigmawort". In: Diekmannshenke, Hajo/ Klein, Josef (eds.): Wörter in der Politik. Opladen: 185-218.

Geeraerts, Dirk/Grondelaers, Stefan/Bakema, Peter (1994): The Structure of Lexical Variation. Meaning, Naming, and Context. Berlin/New York.

Griesbach, Thorsten/Kilian, Jörg (2001): "Sprachkritik als Unwortkritik. Die Aktion 'Unwörter 2000' und die laienlinguistische Wortkritik". Sprachreport 17, H. 3: 11-17.

Harras, Gisela (1991): "Zugänge zu Wortbedeutungen". In: Harras, Gisela/Haß, Ulrike/ Strauß, Gerhard (eds.): Wortbedeutungen und ihre Darstellung im Wörterbuch. Berlin/New York: 3-96.

Hausmann, Franz Josef (1990): "Das Wörterbuch der schweren Wörter". In: Hausmann, Franz Josef/Reichmann, Oskar/Wiegand, Herbert Ernst/Zgusta, Ladislav (eds.): Wörterbücher. Ein internationales Handbuch zur Lexikographie. Zweiter Teilbd. [...]. Berlin/New York: 1206-1210.

Henne, Helmut/Mentrup, Wolfgang (eds.) (1983): Wortschatz und Verständigungsprobleme. Was sind "schwere Wörter" im Deutschen? Jahrbuch 1982 des Instituts für deutsche Sprache. Düsseldorf. 
Henne, Helmut (1987): "Sprache - Normung - Sprachkultur". In: DIN-Mitteilungen 66, Nr. 1: 14-18.

Hjelmslev, Louis (1968): Die Sprache. Eine Einführung. Aus dem Dänischen übersetzt von Otmar Werner. Darmstadt (zuerst dän. 1963).

Hjelmslev, Louis (1974): "Langue und parole". In: ders.: Aufsätze zur Sprachwissenschaft. Stuttgart: 44-55 (zuerst frz. "Langue et parole, 1942).

Kilian, Jörg (1997): Demokratische Sprache zwischen Tradition und Neuanfang. Am Beispiel des Grundrechte-Diskurses 1948/49. Tübingen.

Kilian, Jörg (2000): "'... die Geschichte ist die wahre Kritik jedes Worts' - Fritz Mauthner und die klassische Semasiologie." In: Henne, Helmut/Kaiser, Christine (eds.): Fritz Mauthner Sprache, Literatur, Kritik. Festakt und Symposion zu seinem 150. Geburtstag. Tübingen: 109-131.

Kilian, Jörg (2001a): "Die Ordnung der Wörter in den Köpfen der Sprecher. Zum Terminus Gruppe in Hermann Pauls Sprachtheorie." In: Burkhardt, Armin/Cherubim, Dieter (eds.): Sprache im Leben der Zeit. Beiträge zur Theorie, Analyse und Kritik der deutschen Sprache in Vergangenheit und Gegenwart. Helmut Henne zum 65. Geburtstag. Tübingen: 261281.

Kilian, Jörg (2001b): "Kritische Semantik. Für eine wissenschaftliche Sprachkritik im Spannungsfeld von Sprachtheorie, Sprachnorm, Sprachpraxis". Zeitschrift für germanistische Linguistik 29: 293-318.

Auch unter www.degruyter.de/journals/zgl/2001/pdf/29_293.pdf

Kilian, Jörg (2003a): "Sprachpolitik im Alltag: 'Political Correctness'". In: Neuland, Eva/ Volmert, Johannes (ed.): Sprache und Politik. Seelze. (= Der Deutschunterricht 55, 2003, H. 1) (demnächst).

Kilian, Jörg (2003b): "Vom Elend in der deutschen Sprachnorm. Zum lexikographischen Umgang mit Menschen aus dem Ausland. Prolegomena für eine kritische Semantik im Wörterbuch". In: Liedtke, Frank/Meyer, Paul Georg (eds.): Multikulti light? Einwanderung und Fremdheit im öffentlichen Diskurs (8. Tagung der AG "Sprache in der Politik" in Aachen, Februar 2002). (demnächst).

Maier, Elisabeth (1984): Studien zur Normtheorie und zur Konzeption der Sprachnorm in französischen Wörterbüchern. Frankfurt/M. etc.

Putnam, Hilary (1990): Die Bedeutung von "Bedeutung". Hrsg. und übersetzt von Wolfgang Spohn. 2., durchgesehene Aufl. Frankfurt/M.

Saussure, Ferdinand de (1967): Grundfragen der allgemeinen Sprachwissenschaft. Hrsg. von Charles Bally und Albert Sechehaye [...]. Übers. von Herman Lommel. 2. Aufl. Berlin.

Strauß, Gerhard/Zifonun, Gisela (1984): "Versuch über 'schwere Wörter'. Zur Frage ihrer systembezogenen Bestimmbarkeit." In: Wiegand, Herbert Ernst (ed.): Studien zur neuhochdeutschen Lexikographie IV. Hildesheim/New York: 381-452. (= GL 1-3/83).

Wittgenstein, Ludwig (1999): "Philosophische Untersuchungen". In: ders.: Werkausgabe, Bd. 1, 12. Aufl. Frankfurt/M.: 225-580. 